\title{
Degenerate Roots of Three Transcendental Equations Involving Spherical Bessel Functions
}

\author{
By Robert L. Pexton and Arno D. Steiger
}

Abstract. Roots of the three transcendental equations

$$
\begin{aligned}
j_{l}(\alpha \lambda) y_{l}(\lambda) & =j_{l}(\lambda) y_{l}(\alpha \lambda), \\
{\left[x j_{l}(x)\right]_{x=\alpha \eta}^{\prime}\left[x y_{l}(x)\right]_{x=\eta}^{\prime} } & =\left[x j_{l}(x)\right]_{x=\eta}^{\prime}\left[x y_{l}(x)\right]_{x=\alpha \eta}^{\prime},
\end{aligned}
$$

and

$$
\left[j_{l}(x)\right]_{x=\alpha \mu}^{\prime}\left[y_{l}(x)\right]_{x=\mu}^{\prime}=\left[j_{l}(x)\right]_{x=\mu}^{\prime}\left[y_{l}(x)\right]_{x=\alpha \mu}^{\prime}
$$

that are degenerate for certain values of the parameter $\alpha \in(0,1)$ are presented. The symbols $j_{l}$ and $y_{l}$ denote the spherical Bessel functions of the first and second kind. Root degeneracies are discussed for each equation individually as well as for pairs of equations. Only positive roots are considered, since the equations are invariant under the transformations $\lambda \longrightarrow-\lambda, \eta \rightarrow-\eta$, and $\mu \rightarrow-\mu$. When $l=0$, only the third equation has nontrivial roots. These roots are identical with the roots of the first equation for $l=1$, i.e. $\mu_{0 n}=\lambda_{1 n}(n=1,2, \ldots)$. Various graphs of $\lambda_{l n}, \eta_{l n}$, and $\mu_{l n}$ display root-degeneracies as intersections of curves. Accurate values of degenerate roots with the corresponding values of $\alpha$ are exhibited in tables.

Roots of the third equation for $l=1(1) 15, n=1(1) 30, \alpha=0.1(0.1) 0.7$, together with their minima and associated values of the parameter $\alpha$, are given in the microfiche supplement accompanying this issue. Roots of the first and the second equation and minima of roots of the second equation are published in v. 31 and v. 32 of this journal.

The roots of the first two equations determine the eigenfrequencies of the transverse electric and the transverse magnetic normal modes of an ideal cavity resonator bounded by two concentric spheres $(r=\alpha R$ and $r=R$ ). The roots of the third equation determine the frequencies of the irrotational magnetic eigenfields.

In [1] roots of the transcendental equations

$$
j_{l}(\alpha \lambda) y_{l}(\lambda)=j_{l}(\lambda) y_{l}(\alpha \lambda)
$$

and

$$
\left[x j_{l}(x)\right]_{x=\alpha \eta}^{\prime}\left[x y_{l}(x)\right]_{x=\eta}^{\prime}=\left[x j_{l}(x)\right]_{x=\eta}^{\prime}\left[x y_{l}(x)\right]_{x=\alpha \eta}^{\prime}
$$

where $j_{l}$ and $y_{l}$ denote spherical Bessel functions of the first and second kind, are presented. In [2] these roots are analyzed as functions of the continuous real param-

Received September 1, 1978. $33 \mathrm{~A} 04$.

AMS (MOS) subject classifications (1970). Primary 65 A05; Secondary 65 H05, 78A04,

Key words and phrases. Roots of transcendental equations, spherical Bessel functions, degenerate roots, electromagnetic cavity resonators. 
eter $\alpha$, where $0<\alpha<1$. The roots of (1) and (2) determine the eigenfrequencies of the transverse electric and the transverse magnetic normal modes of an ideal cavity resonator bounded by two concentric spheres $(r=\alpha R$ and $r=R)$. While the normal modes constitute a complete set of eigenvectors for the expansion of an electromagnetic field in the isolated cavity, they no longer are a complete set of basis vectors if the cavity is coupled to a source of electromagnetic energy. In order to obtain such a complete set, it is necessary to add irrotational electric and magnetic multipole fields to the solenoidal fields of the normal modes. The characteristic frequencies of the irrotational electric multipole fields are also determined by Eq. (1). The characteristic frequencies of the irrotational magnetic multipole fields, however, are determined by the roots of the transcendental equation

$$
\left[j_{l}(x)\right]_{x=\alpha \mu}^{\prime}\left[y_{l}(x)\right]_{x=\mu}^{\prime}=\left[j_{l}(x)\right]_{x=\mu}^{\prime}\left[y_{l}(x)\right]_{x=\alpha \mu}^{\prime} .
$$

Roots of Eq. (3) were calculated by the procedure described in [1] and are presented in the microfiche supplement. The ranges of the parameter $\alpha$, of the order $l$ of the spherical Bessel functions, and of the root index $n$ are $\alpha=0.1(0.1) 0.7, l=1(1) 15$, $n=1(1) 30$. The minima of the roots of Eq. (3) are also presented in the microfiche supplement and were computed in the same way as the minima of Eq. (2) were calculated in [2].

Since

$$
\begin{aligned}
& j_{l}\left(z e^{m \pi i}\right)=e^{m l \pi i} j_{l}(z), \\
& y_{l}\left(z e^{m \pi i}\right)=(-1)^{m} e^{m l \pi i} y_{l}(z), \quad(l, m=0,1,2, \ldots)
\end{aligned}
$$

$[3$, p. 439, 10.1.34, 10.1.35], the equations (1), (2), and (3) are invariant under the transformations $\lambda \rightarrow-\lambda, \eta \rightarrow-\eta$, and $\mu \rightarrow-\mu$, respectively. Therefore, only positive roots need be considered. The roots of Eqs. (1), (2), and (3), namely $\lambda_{l n}, \eta_{l n}$, and $\mu_{l n}$, are labelled by the index $n=1,2, \ldots$ such that $\lambda_{l n+1}>\lambda_{l n}, \eta_{l n+1}>\eta_{l n}$, and $\mu_{l n+1}>\mu_{l n}$. For $l=0$, Eqs. (1) and (2) have no nontrivial solutions when $0<\alpha<1$. This is obvious, since for $l=0$ they can both be reduced to the form

$$
\tan (\alpha \lambda)=\tan \lambda
$$

Furthermore, Eq. (3) for $l=0$ is identical with Eq. (1) for $l=1$,

$$
\mu_{0 n}=\lambda_{1 n}, \quad n=1,2, \ldots
$$

For particular values of the parameter $\alpha$ certain roots of the equations (1), (2), and (3) coincide. The following types of root degeneracies are considered

$$
\begin{array}{ll}
\lambda_{l n}(\alpha)=\lambda_{l^{\prime} n^{\prime}}(\alpha), & (l, n) \neq\left(l^{\prime}, n^{\prime}\right), \\
\eta_{l n}(\alpha)=\eta_{l^{\prime} n^{\prime}}(\alpha), & (l, n) \neq\left(l^{\prime}, n^{\prime}\right), \\
\mu_{l n}(\alpha)=\mu_{l^{\prime} n^{\prime}}(\alpha), & (l, n) \neq\left(l^{\prime}, n^{\prime}\right),
\end{array}
$$


and

$$
\begin{aligned}
& \lambda_{l n}(\alpha)=\eta_{l^{\prime} n^{\prime}}(\alpha), \\
& \lambda_{l n}(\alpha)=\mu_{l^{\prime} n^{\prime}}(\alpha), \\
& \eta_{l n}(\alpha)=\mu_{l^{\prime} n^{\prime}}(\alpha)
\end{aligned}
$$

The graphs, Figures 1 through 4, which show the dependence of various roots $\lambda_{l n}, \eta_{l n}$, and $\mu_{l n}$ on the parameter $\alpha$, exhibit as intersections of curves some of the root degeneracies (4) and (5).

Write Eqs. (1), (2), and (3) as

$$
\begin{aligned}
& F_{l}(\alpha, \lambda)=0, \quad \text { where } F_{l}(\alpha, \lambda)=j_{l}(\alpha \lambda) y_{l}(\lambda)-j_{l}(\lambda) y_{l}(\alpha \lambda), \\
& G_{l}(\alpha, \eta)=0, \quad \text { where } G_{l}(\alpha, \eta)=s_{l}(\alpha \eta) t_{l}(\eta)-s_{l}(\eta) t_{l}(\alpha \eta), \\
& s_{l}(x)=x j_{l-1}(x)-l j_{l}(x), \quad t_{l}(x)=x y_{l-1}(x)-l y_{l}(x),
\end{aligned}
$$

and

$$
\begin{gathered}
K_{l}(\alpha, \mu)=0, \quad \text { where } K_{l}(\alpha, \mu)=v_{l}(\alpha \mu) w_{l}(\mu)-v_{l}(\mu) w_{l}(\alpha \mu), \\
v_{l}(x)=j_{l-1}(x)-\frac{l+1}{x} j_{l}(x), \quad w_{l}(x)=y_{l-1}(x)-\frac{l+1}{x} y_{l}(x) .
\end{gathered}
$$

If $p_{l n}$ or $q_{l n}$ represent any one of the roots $\lambda_{l n}, \eta_{l n}$, and $\mu_{l n}$, and if $P_{l}$ or $Q_{l}$ represent any one of the functions $F_{l}, G_{l}$, and $K_{l}$, then the algorithm used to compute degenerate roots can be stated as follows:

Step 1. Input values for $l, n, l^{\prime}, n^{\prime}, \alpha, \Delta \alpha, p_{l n}, q_{l^{\prime} n^{\prime}}, \Delta p_{l n}, \Delta q_{l^{\prime} n^{\prime}}, \epsilon, d d, t$, where $p_{l n}, q_{l^{\prime} n^{\prime}}$ and $\alpha$ are approximations for the desired degenerate root and the associated value of the parameter. The approximations can readily be obtained from the graphs or the root tables.

$$
\begin{aligned}
\epsilon & \text { error tolerance for }\left|p_{l n}-q_{l^{\prime} n^{\prime}}\right| \\
d d & \text { coefficient used to decrease } \Delta \alpha \\
t & \text { determines the sign of } \Delta \alpha . \text { Initially } t=1 .
\end{aligned}
$$

Step 2. With the current value of $\alpha$ compute new values of $p_{l n}$ and $q_{l^{\prime} n^{\prime}}$ such that

$$
P_{l}\left(\alpha, p_{l n}\right)=0 \quad \text { and } \quad Q_{l}\left(\alpha, q_{l^{\prime} n^{\prime}}\right)=0
$$

Step 3. Test the new roots. If $\left|p_{l n}-q_{l^{\prime} n^{\prime}}\right|<\epsilon$, we have our desired degenerate root and associated value $\alpha$.

Step 4. Otherwise, if $\left(p_{l n}-q_{l^{\prime} n^{\prime}}\right) t<0$, then $-t \rightarrow t$ and $d d \Delta \alpha \rightarrow \Delta \alpha$.

Step 5. $\alpha+t \Delta \alpha \rightarrow \alpha$. Return to Step 2 .

A FORTRAN program implementing this algorithm computes values of the degenerate roots (4) and (5) together with the associated values of $\alpha$ to near machine accuracy. Results are presented in Tables 1 through 6 . 


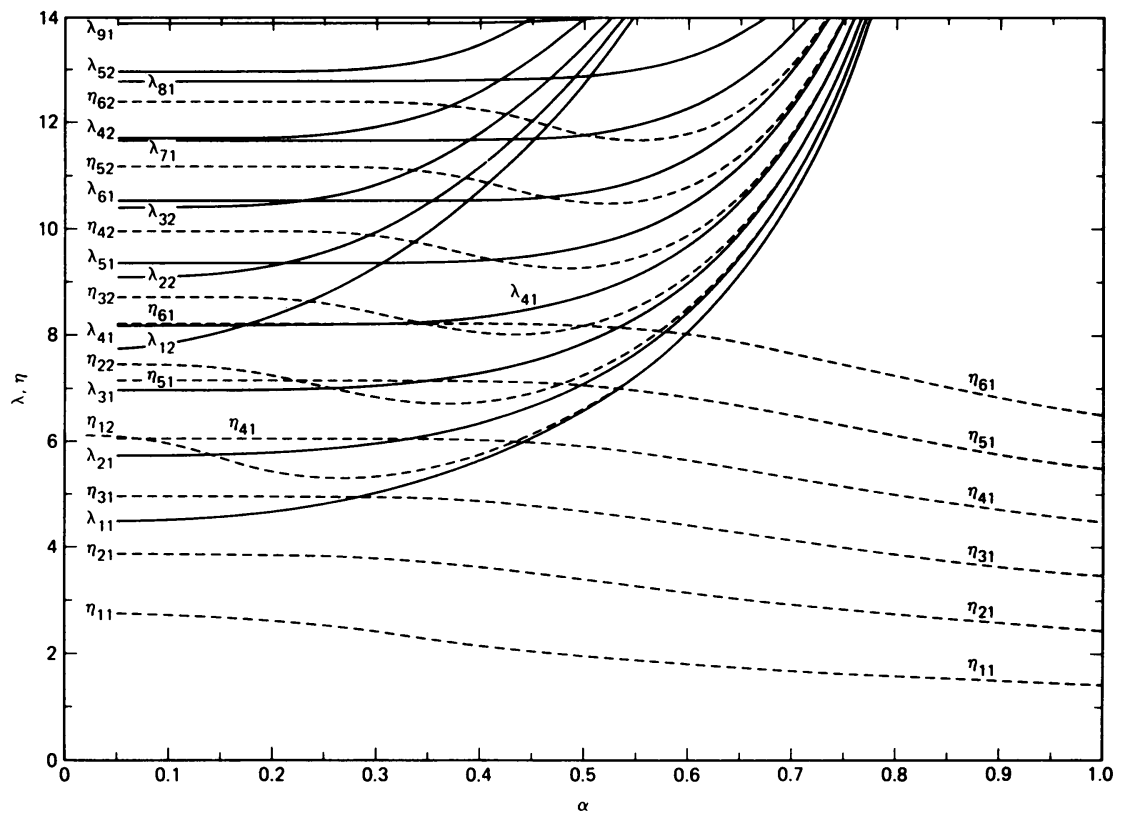

Figure 1

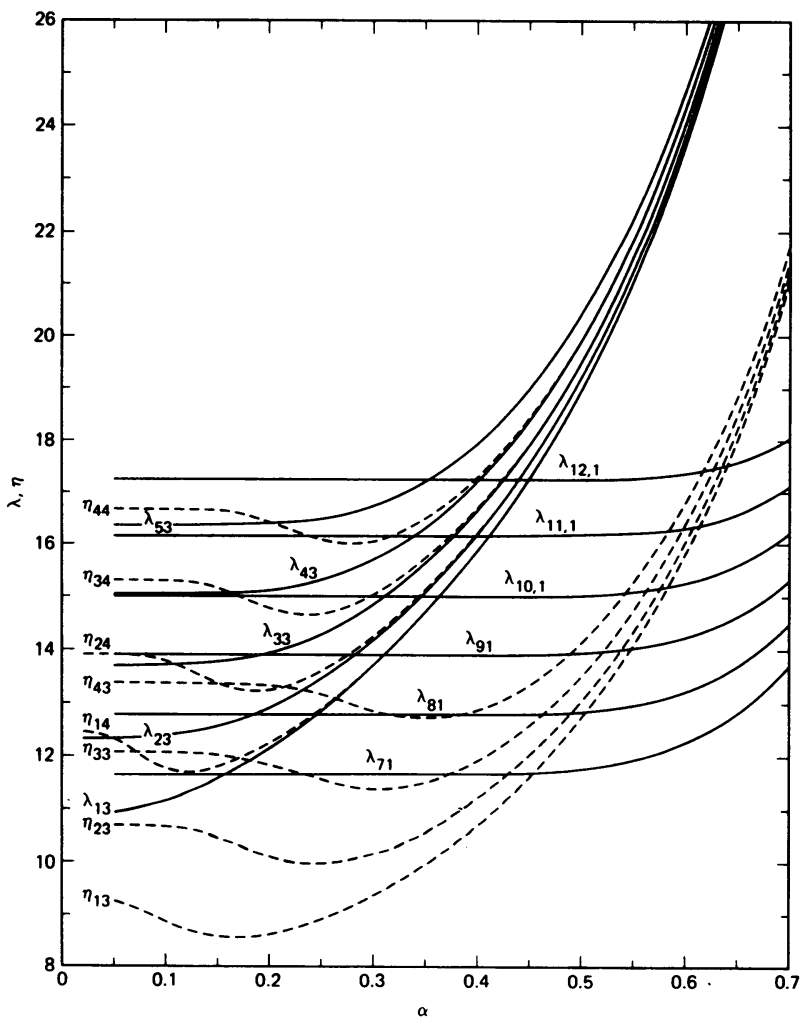

FigURE 2 


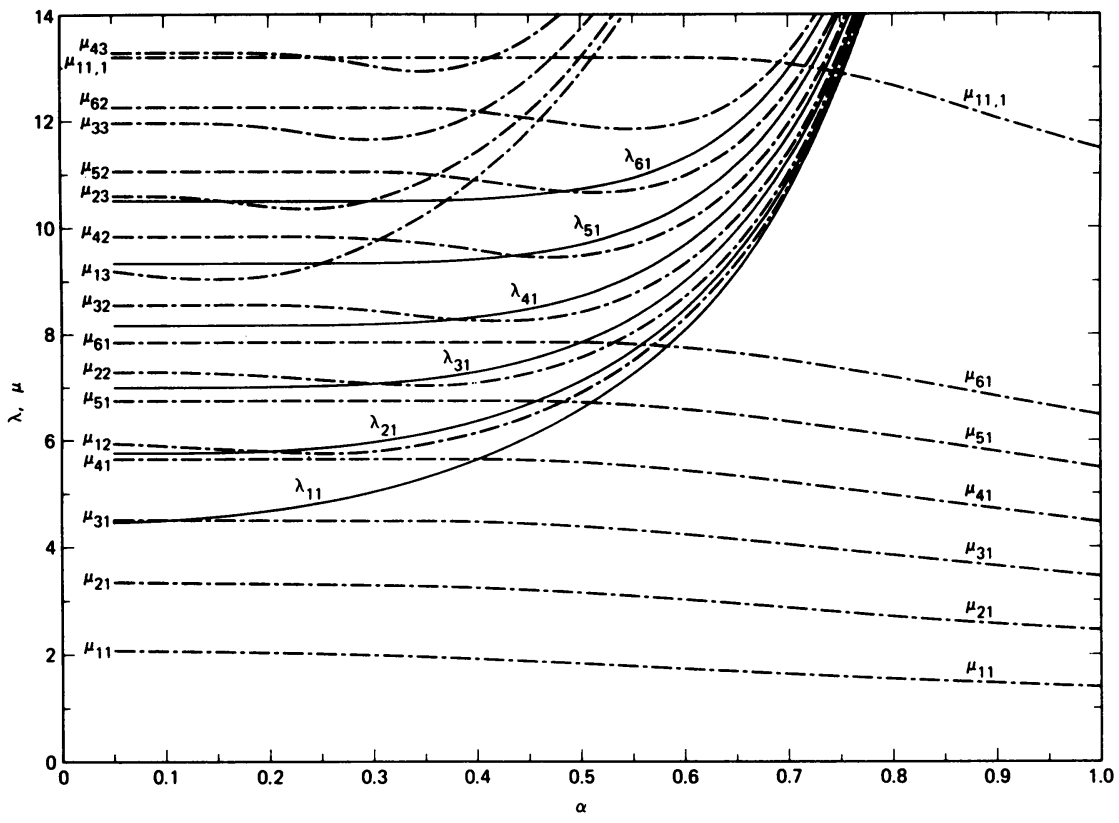

Figure 3

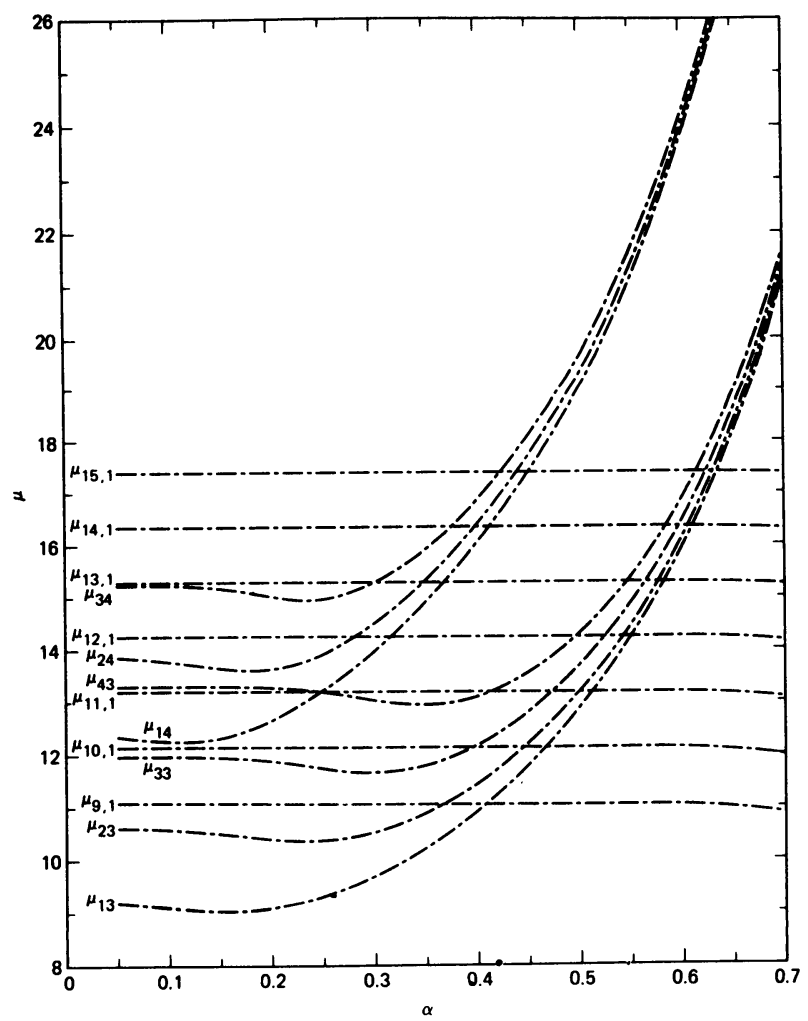

Figure 4 
TABLE 1

\section{$\left(L N L^{\circ} N^{*}\right) \quad$ ALPHA}

$\begin{array}{rrrr}4 & 1 & & 1 \\ 5 & 1 & 1 & 2 \\ 6 & 1 & 1 & 2 \\ 6 & 1 & 2 & 2 \\ 6 & 1 & 3 & 2 \\ 7 & 1 & 1 & 2 \\ 7 & 1 & 1 & 3 \\ 7 & 1 & 2 & 2 \\ 7 & 1 & 3 & 2 \\ 8 & 1 & 1 & 2 \\ 8 & 1 & 1 & 3 \\ 8 & 1 & 2 & 2 \\ 8 & 1 & 3 & 2 \\ 8 & 1 & 4 & 2 \\ 9 & 1 & 1 & 2 \\ 9 & 1 & 1 & 3 \\ 9 & 1 & 2 & 2 \\ 9 & 1 & 2 & 3 \\ 9 & 1 & 3 & 2 \\ 9 & 1 & 3 & 3 \\ 9 & 1 & 4 & 2 \\ 9 & 1 & 5 & 2 \\ 10 & 1 & 1 & 2 \\ 10 & 1 & 1 & 3 \\ 10 & 1 & 2 & 2 \\ 10 & 1 & 2 & 3 \\ 10 & 1 & 3 & 2 \\ 10 & 1 & 3 & 3 \\ 11 & 1 & 1 & 2 \\ 11 & 1 & 1 & 3 \\ 11 & 1 & 2 & 2 \\ 11 & 1 & 2 & 3 \\ 11 & 1 & 3 & 2 \\ 11 & 1 & 3 & 3 \\ 11 & 1 & 4 & 3 \\ 12 & 1 & 1 & 3 \\ 12 & 1 & 2 & 3 \\ 12 & 1 & 3 & 3 \\ 12 & 1 & 4 & 3 \\ 12 & 1 & 5 & 3 \\ & & & \\ 1 & & \end{array}$

$1.7126280679338 \mathrm{E}-01$ 3.0409732823857E-01

$3.8976135002034 \mathrm{E}-01$ $3.5386004528366 \mathrm{E}-01$ 2.2097368533374E-01 $4.5437133153515 \mathrm{E}-01$ $1.5561575902538 \mathrm{E}-01$ 4. $3360736205650 \mathrm{E}-01$ $3.9132959670722 \mathrm{E}-01$ $5.0576934362594 \mathrm{E}-01$ $2.4512007807570 \mathrm{E}-01$ $4.9211925714532 \mathrm{E}-01$ $4.6761080775992 \mathrm{E}-01$ $4.2082222482786 \mathrm{E}-01$ $5.4792997100230 \mathrm{E}-01$ $3.1159812200931 \mathrm{E}-01$ $5.3830422197287 \mathrm{E}-01$ 2.8402501739023E- 1 $5.2197951870556 \mathrm{E}-01$ $1.9978525072569 \mathrm{E}-01$ $4.9509609247442 \mathrm{E}-01$ 4. $4467217849102 \mathrm{E}-01$ $5.8325888352305 \mathrm{E}-01$ 3.6552966752839E-01 $5.7614980031642 \mathrm{E}-01$ 3. $4833291387055 \mathrm{E}-01$ $5.6447336371849 \mathrm{E}-01$ 3. $1393437146020 \mathrm{E}-01$ $6.1334878454385 \mathrm{E}-01$ 4. $1080728016735 \mathrm{E}-01$ 6.0791916366849E-ด1 3.9892636166092E-01 $5.9917971203256 \mathrm{E}-01$ $3.7770573236507 \mathrm{E}-\overline{91}$ $3.3748506326985 \mathrm{E}-01$ 4. $4959836288581 \mathrm{E}-0$ 4. $4089502244773 \mathrm{E}-01$ 4. $2616668360948 \mathrm{E}-01$ 4. $193870378578 E-01$

$3.5606684458354 \mathrm{E}-01$
$\operatorname{LAMBDA}_{L N}=\mathrm{LAMBDA}_{L^{\prime} \mathrm{N}^{\prime}}$

8. $1827583831863 E+00$ 9. $3613355361517 \mathrm{E}+0 \mathrm{Q}$ $1.0532706055389 \mathrm{E}+01$ $1.0520195451432 \mathrm{E}+01$ $1.0512868726504 \mathrm{E}+$. 1 1. $1700001008926 \mathrm{E}+01$ 1. $1657032221077 \mathrm{E}+1$ 1. $1682964958136 E+01$ 1. $1665087032676 \mathrm{E}+01$ $1.2864655267768 \mathrm{E}+01$ $1.2790784560892 \mathrm{E}+01$ $1.2845000200407 \mathrm{PE}+1$ $1.2820563923668 \mathrm{E}+01$ 1. 2798 P $35655857 E+01$ 1. $4027463366049 E+01$ 1. $3915849824674 E+01$ 1. $4006383094531 \mathrm{E}+01$ 1. $3915828212438 E+01$ 1. $3978168461079 E+01$ 1. $3915822621344 E+01$ 1. $3947734449023 E+01$ 1. $3923184183159 \mathrm{E}+01$ 1. $5188915448963 E+01$ 1. $5033586473287 E+01$ 1. 51 ह $149736328 E+01$ 1. $5033518544033 E+91$ 1. $5136868983422 E+\square 1$ 1. $5033476537993 E+01$ 1. $6349335806599 E+01$ 1. $6145080366711 \mathrm{E}+01$ 1. $6327346699072 \mathrm{E}+01$ 1. $61449379>5439 E+01$ 1. $6296054654392 \mathrm{E}+01$ 1. E144810875390E+01 1. $6144750217050 \mathrm{E}+01$ 1. $7251218533827 \mathrm{E}+01$ 1. $7250975357694 \mathrm{E}+01$ 1. $7250719265198 E+01$ 1. $7250534757090 E+01$ 1. $7250460870201 \mathrm{E}+01$

\section{TABLE 2}

\section{$\left(L N L^{\circ} N^{*}\right) \quad$ ALPHF}

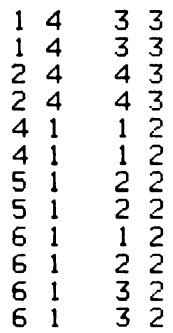
$7.1506742584463 \mathrm{E}-02$ $1.7269587060611 \mathrm{E}-01$ $1.4739100984938 \mathrm{E}-01$ $2.1447582237830 \mathrm{E}-01$ 7.0010866660159E-פ2 4.3458767568285E- $2.2766429824463 \mathrm{E}-\overline{1} 1$ 4.775299736426PE- 1 $5.9706171548852 \mathrm{E}-01$ $5.7280039842735 \mathrm{E}-01$ $3.465034236 .0255 \mathrm{E}-01$ $5.0211244439851 \mathrm{E}-\overline{1} 1$

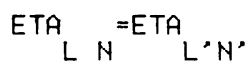

$1.2063302630523 \mathrm{E}+01$ 1. $1971485186709 E+01$ $1.3375996312163 \mathrm{E}+01$ 1. $3301666048358 \mathrm{E}+01$ 6. $0619493542981 \mathrm{E}+01$ 6. $0030517595060 \mathrm{E}+00$ $7.1401934217484 \mathrm{E}+00$ 7. $.8874487501519 \mathrm{E}+00$ 8. $176551250788 \mathrm{E}+00$ 8. $0738795809855 E+00$ 8.2102063139689E+00 $8.1713407895880 \mathrm{E}+00$ 
TABLE 3

\begin{tabular}{|c|c|c|}
\hline LN & $\mathrm{L}^{*} \mathrm{H}^{*}$ ) & ALPHA \\
\hline 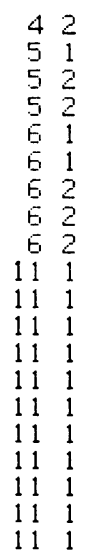 & $\begin{array}{ll}1 & 3 \\
1 & 2 \\
1 & 3 \\
2 & 3 \\
1 & 2 \\
2 & 2 \\
1 & 3 \\
2 & 3 \\
3 & 3 \\
1 & 2 \\
1 & 3 \\
2 & 2 \\
2 & 3 \\
3 & 2 \\
3 & 3 \\
4 & 2 \\
4 & 3 \\
4 & 3 \\
5 & 2 \\
6 & 2\end{array}$ & 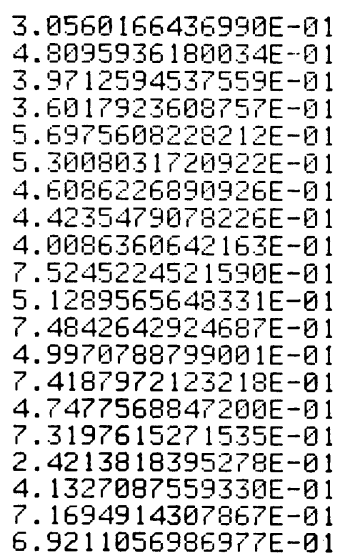 \\
\hline
\end{tabular}

$M U L N U_{L \cdot N}$.

9.7768178761880E+ +09

6.7286219288186E+0日

$1.0907437280180 \mathrm{E}+01$

$1.0985809391387 \mathrm{E}+01$

T. $7799021272269 \mathrm{E}+90$

7.8151404673729E+

$1.2027472118634 \mathrm{E}+191$

$1.2082141526461 \mathrm{E}+01$

$1.2183767505963 \mathrm{E}+01$

$1.2900218460577 E+01$

$1.3201822683999 \mathrm{E}+01$

$1.2916571602170 \mathrm{E}+01$

$1.3202131098528 \mathrm{E}+01$

1. $2942076321558 \mathrm{E}+01$

$1.3202436363935 E+01$

1. $2978024567707 E+01$

1. $3202620396698 \mathrm{E}+01$

$1.3202608622369 \mathrm{E}+01$

$1.3026357464333 E+01$

1. $3089897269223 \mathrm{E}+01$

\section{TABLE 4}
$(L N$
$\left.L^{\prime} N^{*}\right)$
ALPHA

ETA

$L_{N}=L A M B D A$ L'N"

$1.3689030947367 \mathrm{E}-01$

$2.5490599344310 \mathrm{E}-01$

$2.8204263961059 \mathrm{E}-01$

$2.3275232200162 \mathrm{E}-01$

$3.4191635465948 \mathrm{E}-01$

4. $4340090404323 \mathrm{E}-01$

$3.3126377223292 \mathrm{E}-01$

$3.3538474533576 \mathrm{E}-01$

2. $9128555571746 \mathrm{E}-01$

4. $0829201719491 \mathrm{E}-01$

5.34677ด $1345668 \mathrm{E}-01$

4. $9554033050096 \mathrm{E}-01$

$3.5099931773671 \mathrm{E}-01$

4. $0869156889741 \mathrm{E}-01$

3.8640362512342E-01

$3.3614281815884 \mathrm{E}-01$

4. $6069883942953 \mathrm{E}-01$

5. $9788204763506 \mathrm{E}-01$

1. $7571383663424 \mathrm{E}-\mathrm{b} 1$

$5.7691251645373 \mathrm{E}-01$

5. $3278531610879 E-01$

$3.1781767672858 E-01$

4. EE $3765809816 \mathrm{E}-\mathrm{D}_{\mathrm{i}}$

4.5195326790701F-01

4. $2624725825721 \mathrm{E}-01$

$3.7206387075334 \mathrm{E}-01$

$5.032503450341 \mathrm{EE}-01$

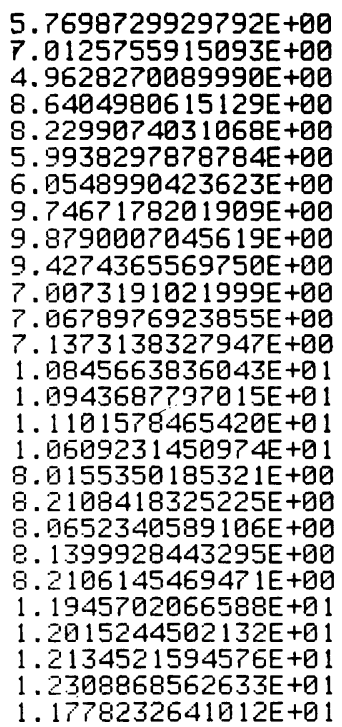


TABLE 5

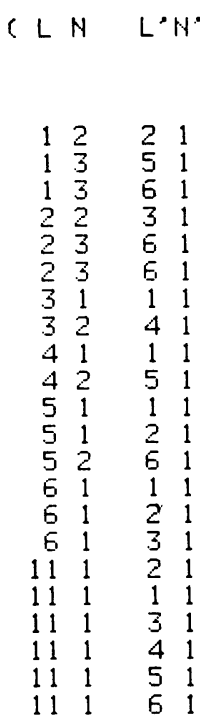

ALFHA

$1.9772652403568 E-01$ $2.5328228275670 \mathrm{E}-01$ $3.7047960270123 \mathrm{E}-01$ $3.0582883643917 \mathrm{Z}-01$ 1. $5518972152495 E-01$ $2.9717742615205 E-01$ 8.8968075131997E-02 $3.8473298267519 \mathrm{E}-01$ $3.9915602451889 \mathrm{E}-\mathrm{Q} 1$ 4. $4493361027329 E-01$ $5.1154937581843 \mathrm{E}-01$ $4.57>1229756763 E-01$ 4. $9257661295007 \mathrm{E}-01$ $5.8381749833796 \mathrm{E}-\overline{\mathrm{V}} 1$ 5. $5807931870672 \mathrm{E}-01$ $4.9833756415319 \mathrm{E}-01$ 7. $5063221509065 \mathrm{E}-0$ $7.5437582202699 \mathrm{E}-01$ $7.4461769841400 \mathrm{E}-01$ $7.3571932123530 \mathrm{E}-01$ $7.2278727362398 \mathrm{E}-01$ 7. $0343701937221 \mathrm{E}-01$

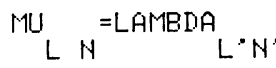

$5.7978760571995 E+00$ $9.3567654234745 E+00$ 1. $0524710853371 \mathrm{E}+01$ $7.0585791986358 \mathrm{E}+00$ 1. $0512835828998 \mathrm{E}+01$ 1. $0513921759296 \mathrm{E}+01$ $4.5140980452001 \mathrm{E}+00$ 8. $2885288005756 \mathrm{E}+00$ $5.6327241415465 E+00$ $9.4955108800748 \mathrm{E}+00$ E. $7092762876190 \mathrm{E}+00$ E. $7385473904137 \mathrm{E}+00$ 1. $0684757078089 E+01$ $7.7625837496334 \mathrm{E}+0 \mathrm{Q}$ $7.7922454482169 E+00$ $7.8317911293936 E+00$ 1. $29076737>7424 E+01$ 1. $2892228576026 \mathrm{E}+01$ 1. $2931575676039 \mathrm{E}+01$ 1. $2964815574344 \mathrm{E}+01$ 1. $3008476135871 \mathrm{E}+01$ 1. $3063400003542 \mathrm{E}+01$

TABLE 6

\section{( LN L'N') \\ ALPHA}

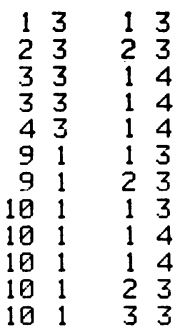

$6.3289226469119 \mathrm{E}-02$ $1.3291182914516 \mathrm{E}-01$ 7.8698037003981E-02 $1.6847453074461 \mathrm{E}-01$ $2.6549491005295 \mathrm{E}-01$ $4.2113462436406 \mathrm{E}-01$ $3.9090746881455 \mathrm{E}-01$ $4.7479710312284 \mathrm{E}-01$ $6.5333002137485 E-02$ $1.9041480082058 \mathrm{E}-01$ $4.5687185169372 E-01$ 4. $1866597226675 \mathrm{E}-01$

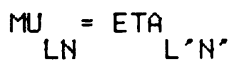

$9.1761179764685 \mathrm{E}+00$ 1. $0557617071159 E+01$ 1. $1972438464397 \mathrm{E}+\square 1$ 1. $1935272056718 \mathrm{E}+\square 1$ $1.3139594547296 \mathrm{E}+\square 1$ 1. $1079274136952 \mathrm{E}+01$ 1. $1079376976673 \mathrm{E}+01$ $1.2142770261848 \mathrm{E}+01$ $1.2143204100943 \mathrm{E}+01$ $1.2143204109935 E+01$ $1.2142984799306 \mathrm{E}+01$ 1. $2143159104602 E+01$

University of California

Lawrence Livermore Laboratory

Livermore, California 94550

1. ROBERT L. PEXTON \& ARNO D. STEIGER, "Roots of two transcendental equations involving spherical Bessel functions," Math. Comp., v. 31, 1977, pp. 752-753.

2. ROBERT L. PEXTON \& ARNO D. STEIGER, "Roots of two transcendental equations as functions of a continuous real parameter," Math. Comp., v. 32, 1978, pp. 511-518.

3. M. ABRAMOWITZ \& I. A. STEGUN (Editors), Handbook of Mathematical Functions with Formulas, Graphs and Mathematical Tables, 3rd ed., Nat. Bur. Standards, Appl. Math. Ser., No. 55, Superintendent of Documents, U. S. Government Printing Office, Washington, D. C., 1965. MR 31 \#1400. 